DOI: https://doi.org/10.46296/rc.v5i9.0039

\title{
Marketing Político 2.0: Estrategias de storytelling a través de TikTok de los dos candidatos a presidente del Ecuador 2021
}

\section{Political Marketing 2.0: Storytelling strategies through Tik Tok of two candidates for president of Ecuador 2021}

\author{
Suárez-Tomalá Geovanny \\ Universidad Estatal Península de Santa Elena. Santa Elena, Ecuador. \\ Correo: geosum.00@gmail.com \\ ORCID ID: https://orcid.org/0000-0001-9189-5329 \\ Cochea-Panchana Gerzon \\ Universidad Estatal Península de Santa Elena. Santa Elena, Ecuador. \\ Correo: gcochea@upse.edu.ec \\ ORCID ID: https://orcid.org/0000-0001-6863-4999
}

\begin{abstract}
RESUMEN
El uso de las redes sociales como herramientas de comunicación política es una tendencia que se evidenció con mucha mayor fuerza en Ecuador durante las campañas presidenciales del 2021, las cuales se dieron en medio de la crisis por COVID-19. En dicho marco, el presente documento investigativo presenta como objetivo principal el análisis del uso del discurso como parte de la estrategia de storytelling, implementado por los candidatos Guillermo Lasso y Andrés Arauz en la red social TikTok. La metodología de la investigación fue de carácter mixta, usando como técnica de recolección de datos la observación de los videos subidos por los candidatos a la plataforma, revisando las principales características discursivas que estos poseían. Entre los principales hallazgos se evidencia que el alcance general es similar al de otras redes, además, a pesar de que las temáticas discursivas principales son asertivas, es poco probable definir con exactitud un tipo de discurso que resulte efectivo, pues cada video posee un alcance distinto.
\end{abstract}

Palabras claves: comunicación política, discurso, storytelling, marketing 2.0, tiktok.

\begin{abstract}
The use of social networks as tools for political communication is a trend that became much more evident in Ecuador during the 2021 presidential campaigns, which took place in the midst of the COVID-19 crisis. Within this framework, the main objective of this research paper is to analyze the use of discourse as part of the storytelling strategy implemented by candidates Guillermo Lasso and Andrés Arauz in the social network TikTok. The research methodology was of a mixed nature, using as a data collection technique the observation of the videos uploaded by the candidates to the platform, reviewing the main discursive characteristics they possessed. Among the main findings, it is evident that the general scope is similar to that of other networks, in addition, although the main discursive themes are assertive, it is unlikely to accurately define a type of discourse that is effective, since each video has a different scope.
\end{abstract}

Keywords: political communication, speech, storytelling, marketing 2.0, tiktok.

Información del manuscrito:

Fecha de recepción: 01 de diciembre de 2021.

Fecha de aceptación: 27 de diciembre de 2021.

Fecha de publicación: 06 de enero de 2022. 


\section{INTRODUCCIÓN}

La comunicación política es un elemento que ha enmarcado el desarrollo de la sociedad, pues es gracias a esta que el ser humano ha sido capaz de organizar su nación y territorio, usándola como medio para informar a los ciudadanos acerca de sus actividades [1], y convencerlos así de sus ideologías de agrupación política.

Inicialmente, para ejercerla se usaron medios tradicionales, sin embargo, con el pasar de los años, debido a la evolución de la tecnología y las necesidades de la sociedad, surgen nuevas formas y herramientas de comunicación. Es así, que todos aquellos sectores en que la comunicación se encuentra inmersa, han tenido que adaptarse a las nuevas tendencias.

Las anteriormente mencionadas, son consideradas las Nuevas Tecnologías de la Información y Comunicación (NTIC), cuyo objetivo principal es lograr la personalización del mensaje y la interacción entre emisor-receptor, y tal como menciona Arbeláez [2], estas se han transformado, pasando de formar parte de medios complementarios a constituirse como medios principales.

Entre las nuevas tecnologías se encuentra el surgimiento de las redes sociales, mismas que, formando parte de la Web 2.0, "han desarrollado competencias para editar, producir y compartir, de forma interactiva, recursos multimedia, creando de este modo narrativas y vivencias de aprendizaje transmedia" [3], y gracias a su rápido crecimiento de usuarios y aceptación por la audiencia, se convirtieron en fuentes necesarias para el intercambio de información entre usuarios. [4]

En este nuevo proceso de comunicación que se genera en internet, el consumidor es quien tiene la decisión de acceder o no al mensaje, obteniendo un poder que lo lleva a tener actitudes más rigurosas, reflexivas y eficaces que definan la relación individuo-sociedad. [5]

Dentro de la comunicación política, el uso de las NTIC se evidencia a través del hecho de que candidatos a puestos políticos han optado por usarlas como parte de sus campañas, esto debido a factores como el bajo costo de inversión por publicación, el alcance, el compromiso, el público y la analítica. [6] 
Uno de los países pioneros en el uso del marketing político en internet es Estados Unidos, pues desde el año de 1998 se empleaba el correo electrónico para compartir información presidencial a los ciudadanos. Sin embargo, el caso más conocido es el de Barack Obama, quien, en 2007, usó páginas webs y YouTube para acercarse a la audiencia. Una campaña que fue elogiada por medios internacionales, misma que estuvo al mando de Chris Hudges, cofundador de Facebook.

De este modo, el modelo anterior evidenció un cambio dentro de la forma en que se percibe la comunicación política en internet. Para que una campaña en internet funcione debería ser de fácil acceso para la audiencia, y le permita a esta interactuar, logrando escuchar a los ciudadanos a través de estos medios [7], es decir, no basta con subir contenido, sino verificar que haya sido recibido por los usuarios y así compartir sus opiniones y comentarios.

En Ecuador, el uso de herramientas como redes sociales para la transmisión de información política se mantuvo fuera del margen de prioridades durante la última década. No obstante, fue en el año 2013 , en que varios partidos políticos hicieron uso de ellas para elaborar mensajes a través de Facebook y Twitter.

Entre esos partidos se encuentra Alianza PAIS, mismo que Buchelli [8] realizó un análisis, donde determinó que la práctica de revisar información política en internet es ejercida en proporción mínima. Además, menciona que no todos los partidos políticos se preparaban con estrategias de comunicación 2.0, y de esta forma incentivó a los políticos a hacerlo.

A pesar de aquello, no fue hasta el año 2020 en que el uso de las redes sociales se tornó fundamental. La promoción o difusión de contenidos políticos por las Elecciones 2021 dejaron de ser únicamente tradicionales, como las reuniones con comunidades, campañas en televisión y radios [1], sino que debido a la pandemia por COVID-19 y la declaratoria de estado, los candidatos se vieron forzados a usar estrategias digitales para llegar a una mayor cantidad de audiencia, a aquella que por distintas razones no podían acompañarlos en las cruzadas por el territorio ecuatoriano. 
Es aquí donde se hace uso del marketing político 2.0, puesto a que "el electorado recurre a internet para organizarse y participar masivamente en las campañas políticas de sus candidatos que, mediante esta modalidad, buscan ganar las elecciones" [7].

Si bien, en la lista de redes sociales mayormente usadas por los candidatos para transmitir sus campañas se encuentran Facebook, Twitter, Instagram y YouTube, existe una que a inicios de la pandemia ganó popularidad, generando entre enero y marzo de 2020 dos mil millones de usuarios alrededor del mundo [9], y por tal motivo fue usada como estrategia de comunicación para hacer marketing político, la aplicación TikTok.

TikTok es una plataforma en la que inicialmente se compartían videos de lip sync, retos y bailes, no obstante, en la actualidad "llegaron para incursionar de buena o mala manera el mundo de la política" [10].

Debido a su uso como fin de comunicación y marketing político, se ha generado un gran debate ideológico entre politólogos y comunicadores, quienes afirman que su uso es sinónimo de llamar la atención [11], y que su contenido debe estar direccionado a una conversación digital de impacto. [10]

En nuestro país, entre los candidatos que hicieron uso de esta plataforma para transmitir información en sus campañas fueron: Andrés Arauz, Guillermo Lasso, Xavier Hervas y Yaku Pérez. Del grupo mencionado, los dos primeros pasaron a una segunda vuelta electoral.

En dicho marco, el presente artículo presenta un estudio que busca determinar el uso del discurso como parte de la estrategia de storytelling empleada por los candidatos a segunda vuelta electoral para la presidencia de Ecuador 2021, identificando cuáles son aquellos temas que tienen mayor relevancia en las historias emitidas.

\subsection{Estrategia digital de storytelling}

Una estrategia hace referencia al conjunto de procesos, lineamientos o pasos a seguir para alcanzar un objetivo en específico [12]-[13], en este caso, sería el conjunto de recursos comunicativos empleados que permiten alcanzar resultados convenientes para la organización. [14] 
Es importante mencionar que, una estrategia digital debe tener tres aspectos esenciales, mismos que Ugarte [15] define de la siguiente forma:

1. El discurso: relatos de individuos o pequeños grupos que transforman la realidad con voluntad. Cada activista debe estar convencido de lo que dice, de lo que comunica.

2. Las herramientas: instrumentos que hagan visible el hacking social, activistas que a través de redes logran desarrollar su protesta social.

3. La visibilidad: las estrategias deben estar pensadas para que, en pequeños gestos, la ciudadanía pueda reconocerse entre otros como ellos.

El elemento de estudio para la presente investigación fue el discurso. Fog et al. [16] mencionan varios elementos importantes que forman parte del discurso. Entre ellos se encuentran: el mensaje, contenido informativo que se es presentado; los personajes, aquellos recursos humanos que aparecen en el video y con quienes los usuarios mantienen o establecen una conexión; y el argumento.

Donstrup [17], afirma que el mensaje político debe dividirse en cuatro variables en cuanto al análisis de su contenido: positivo, negativo, neutro y reactivo. Sin embargo, para la presente investigación los ejes se alteraron debido a que estos deben adaptarse a la red social que se pretende analizar. De esta forma, las variables a identificar fueron: contenido asertivo, contenido negativo, contenido informativo y contenido reactivo.

Tabla 1. Clasificación del mensaje por su contenido. Elaboración propia a partir de conceptos de Donstrup.

\begin{tabular}{|c|l|}
\hline $\begin{array}{c}\text { Tipo de } \\
\text { Contenido }\end{array}$ & \multicolumn{1}{|c|}{ Breve descripción } \\
\hline Asertivo & $\begin{array}{l}\text { Presentan ideas de cambio, alabanzas sobre sí mismo y no } \\
\text { menciona al oponente. }\end{array}$ \\
\hline Negativo & Atacan al oponente sin hablar positivamente de sí mismos. \\
\hline Informativo & El único fin es informar acerca de alguna situación, evento. \\
\hline Reactivo & $\begin{array}{l}\text { Mencionan cualidades positivas del emisor y descalifican al } \\
\text { oponente. }\end{array}$ \\
\hline
\end{tabular}


Además, la historia contada debe seguir una serie de condicionantes en cuanto a su estructura y su argumento [18].

Gráfico 1. Estructura de una historia. Elaboración propia a partir de conceptos de Martín.

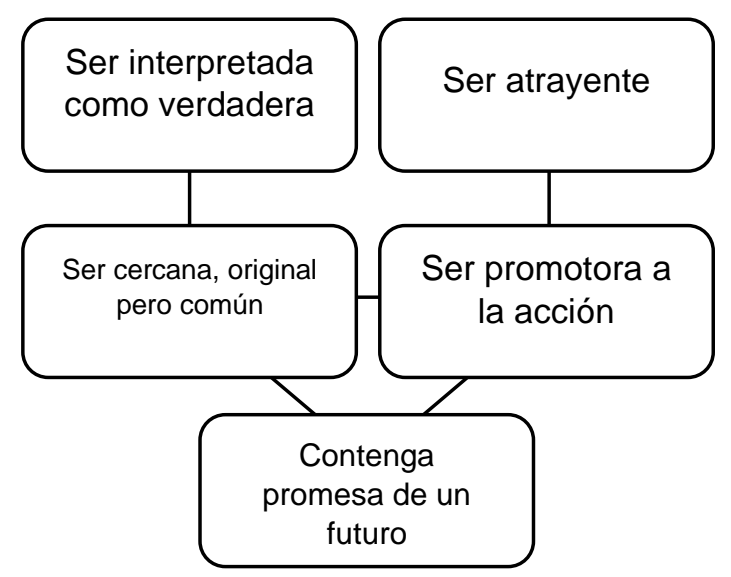

Estas tablas, figuras, y sus conceptos, aportaron de manera esencial para la elaboración de este proyecto, pues ayudaron a definir, jerarquizar y clasificar el contenido presente en las historias contadas por Lasso y Arauz en la red social TikTok.

\section{MATERIALES Y MÉTODOS}

El enfoque de esta investigación fue mixto, se recogieron datos numéricos que proporciona la red social TikTok, y se realizó un análisis exhaustivo acerca del storytelling de cada video. Es así, que se realizó una recolección y análisis de datos cuantitativos y cualitativos, logrando una comprensión mayor del fenómeno estudiado. [19]

Los datos recolectados pertenecen al contenido emitido en las cuentas oficiales de TikTok de Guillermo Lasso, por la alianza CREO - PSC, y Andrés Arauz, por Unión por la Esperanza, mismos que pasaron a una segunda vuelta electoral. El registro se llevó a cabo con datos desde el 21 de febrero, fecha en que se oficializaron los dos candidatos que volverían a participar en el proceso electoral, hasta el 11 de abril, fecha en que fueron las elecciones presidenciales.

El total de videos subidos por cada candidato durante el periodo de estudio es el siguiente: 
Revista Científica Arbitrada de Investigación en Comunicación, Marketing y Empresa REICOMUNICAR. Vol. 5, Núm. 9 (ene - jun 2022) ISSN: 2737-6354.

Marketing Político 2.0: Estrategias de storytelling a través de TikTok de los dos candidatos a presidente del Ecuador 2021.

Tabla 2. Total, de videos subidos por cada candidato desde el 21 FEB - 11 ABR. Elaboración propia

\begin{tabular}{|c|c|}
\hline Candidato & \# Videos \\
\hline Andrés Arauz & 40 \\
\hline Guillermo Lasso & 37 \\
\hline Total & 77 \\
\hline
\end{tabular}

De los 77 videos, se seleccionaron 50 de manera aleatoria, dividiendo 25 videos de Guillermo Lasso y 25 videos de Andrés Arauz, puesto a que el total de videos no era igualitario, entonces se procedió a hacer un equilibrio en el objeto de estudio. Estos videos serán seleccionados de manera aleatoria a través de la página WinEpi, que permite hacer una selección al azar de individuos de una población.

La ficha de observación se realizó basándose en conceptos de Fog et al. [16] y otros autores [17]-[18], con el fin de estudiar, analizar y jerarquizar la tipología del mensaje político difundido en TikTok, además de recolectar datos de interacción y de observación.

El instrumento de investigación fue revisado y validado por expertos para su uso, quedando divido en cinco secciones.

Tabla 3. Secciones de la ficha de observación. Elaboración propia

Sección

\begin{tabular}{|c|c|}
\hline Registro de datos & Esta sección permitirá registrar datos numéricos que ofrece la red \\
social: likes y reproducciones.
\end{tabular}




\section{DISCUSIÓN Y RESULTADOS}

A través del análisis y jerarquización de datos, según las variables y dimensiones consideradas, se evidencia diferentes estrategias de discurso que han hecho uso los partidos políticos que pasaron a una segunda vuelta electoral. En primera instancia, a través de los datos que proporciona la red social TikTok, se demuestra que la cantidad de reacciones (likes) por video es considerable (Gráfico 2). Se logra constatar que para el candidato Lasso, la cantidad de likes en sus videos mayormente varía entre los 100 mil y 500 mil; mientras que para el candidato Arauz, la cantidad de likes varía con mayor frecuencia entre 10 mil y 100 mil.

Gráfico 2. Cantidad de likes por video. Elaboración propia a partir de datos de la ficha de observación.

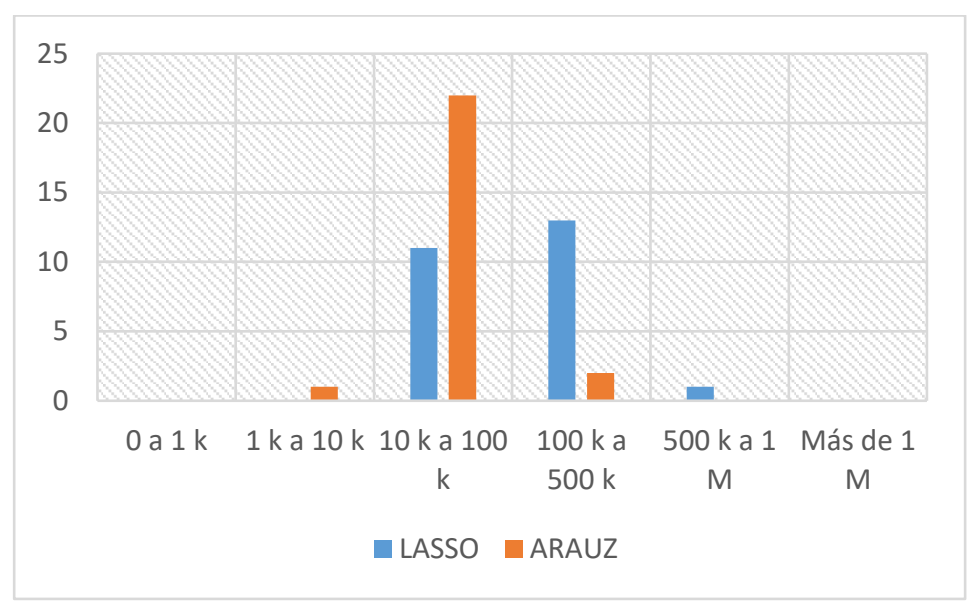

Con respecto al alcance de los videos (Gráfico 3), la mayor frecuencia de visualizaciones para el candidato Lasso es de más de 1 millón, mientras que para el candidato Arauz la frecuencia es entre 100 mil y 500 mil. Esto tiene correlación con la cantidad de likes, pues suben los porcentajes. Se puede deducir que, por cada 100 visualizaciones generadas en un video, surge 1 like. Es así, que se puede afirmar que el candidato con mayor recepción de interacciones de la audiencia en la red social de estudio es el candidato Lasso, pues sus videos poseen un gran alcance, datos evidenciados a través de la cantidad de likes y reproducciones que más del $50 \%$ de sus videos poseen. 
Gráfico 3. Cantidad de reproducciones por video. Elaboración propia a partir de datos de la ficha de observación.

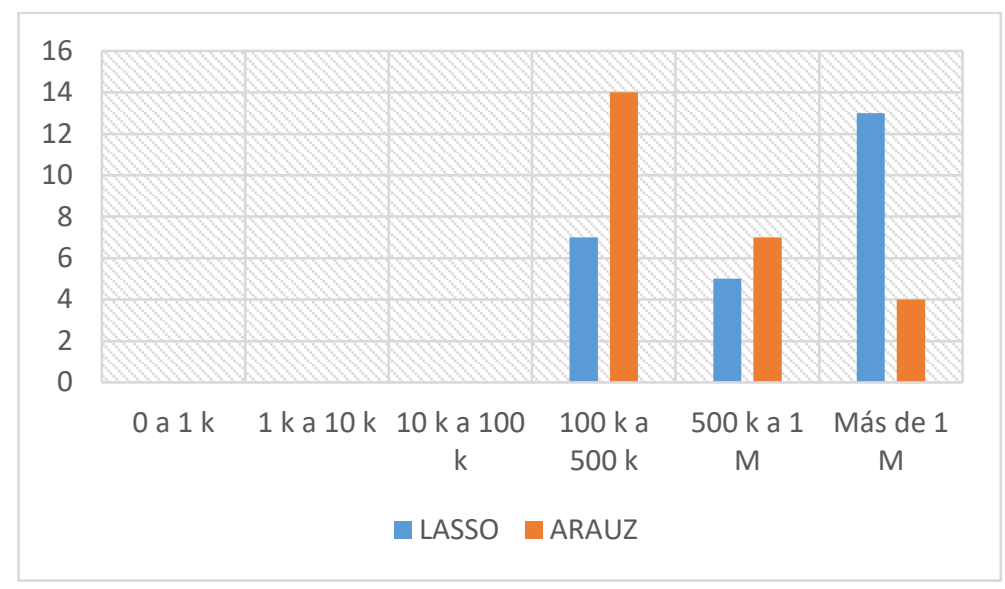

En relación a la tipología del mensaje, la que mayormente ha sido usada en los dos casos durante el proceso de campaña electoral por segunda vuelta (Gráfico 4) ha sido la asertiva, superando el $50 \%$ de los videos subidos por el candidato Lasso (52\%) y el candidato Arauz (60 \%). De igual forma, se puede observar que quien tiene mayor cantidad de mensajes con contenido negativo de entre los dos candidatos es Lasso (20\%). No obstante, este tipo de contenido queda en tercer lugar, y quien lo precede es el de tipo informativo en ambos casos, $28 \%$ para el candidato Lasso, y 32 \% para el candidato Arauz. Esto además queda evidenciado en la observación anecdótica, ya que los videos que fueron categorizados como informativos son aquellos que únicamente tenían como propósito informar sobre alguna actividad de campaña ya realizada, o que se ejecutaría en un futuro.

Gráfico 4. Tipología del mensaje de los videos de Lasso y Arauz en TikTok. Elaboración propia a partir de datos de la ficha de observación.

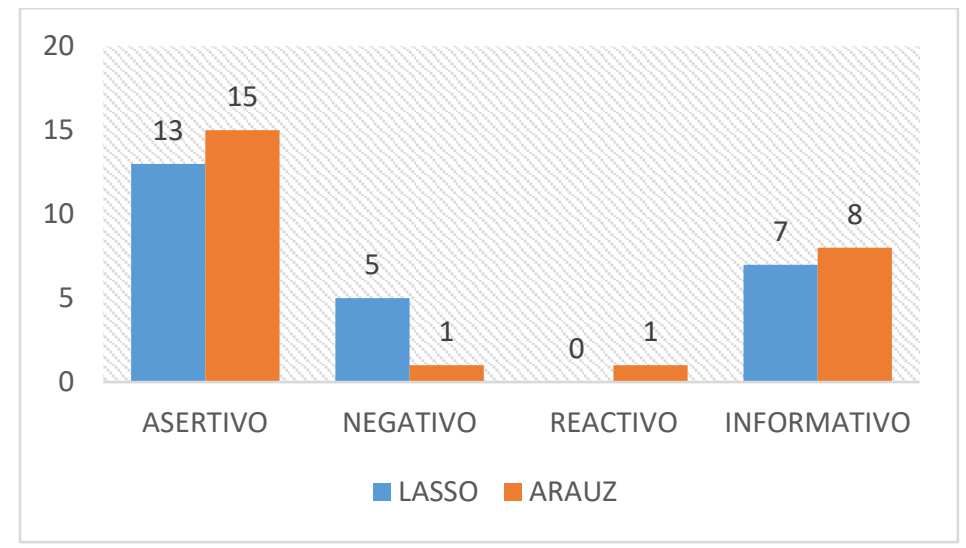


El único candidato que realizó un mensaje reactivo, el cual consistía en mencionar cualidades negativas del candidato opositor, y mencionar cualidades positivas del candidato emisor del mensaje, fue Arauz. Aquel video es particular, pues menciona hechos del pasado que, según Arauz, Lasso estuvo involucrado. Es así, que, posterior de hablar negativamente de él, indica que en 'su gobierno' no realizarán aquello. Además, este video fue el que mayor visualización obtuvo $(1,5 \mathrm{M})$.

Dentro de la tipología asertiva (Gráfico 5), el candidato por CREO mantiene un número igualitario en cuando a videos con temática de intimidad (12\%) y esperanza (12\%), dentro de estos se encuentra, además, el video con mayor cantidad de visualizaciones (7,8 M), un video en el que, junto a su esposa, preparan un plato tradicional ecuatoriano, además de que este va de la mano con una canción y trend del momento.

Por otro lado, respecto a los mensajes negativos, el que mayor frecuencia tiene es el de tipología adversario (12\%), ya que, el candidato en mención, usaba videos del opositor para mencionar cualidades o acciones negativas, pero sin caer en la auto exaltación. Es justamente dentro de esta categoría, que se encuentra el video que generó mayor reacción de la audiencia, pues los likes superan el medio millón (543,0 k) y cerca de 20 mil comentarios (19,3 k). Sin embargo, no es el más visto, ya que es superado por el video que realizó junto a su esposa, mencionado anteriormente.

Gráfico 5. Cantidad y clasificación de mensajes con tipología asertiva y negativa del candidato Lasso. Elaboración propia a partir de datos de la ficha de observación.

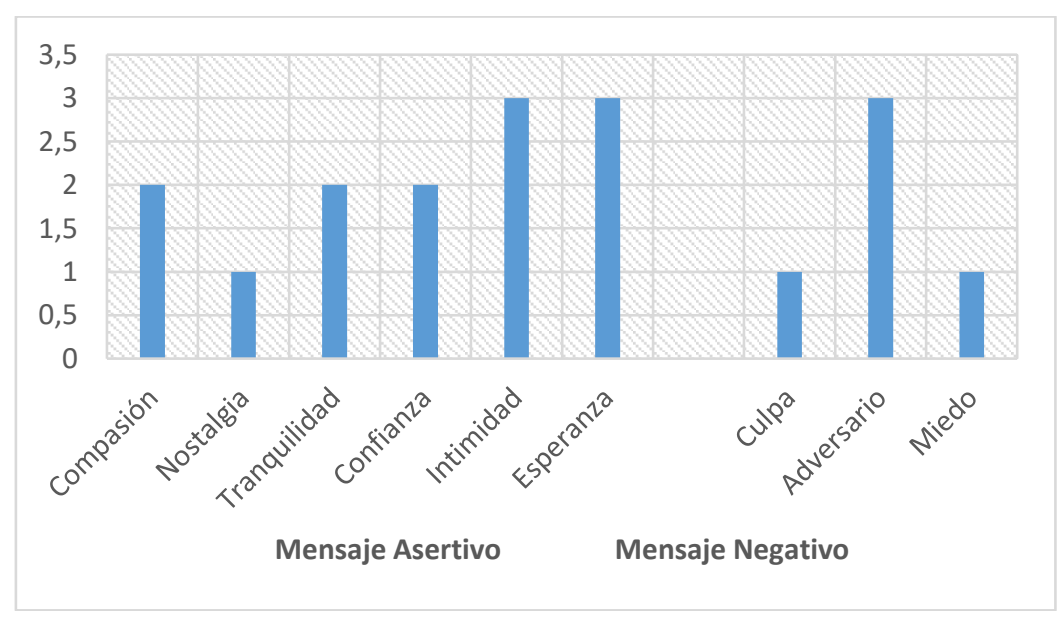


En cambio, los datos de las temáticas principales usadas en videos del candidato por Unión por la Esperanza (Gráfico 6) son: en la asertiva, la tipología esperanza (24\%), los cuales a través de la observación podemos detallar que este candidato presentaba propuestas para el cambio en sus videos, acompañado de sugerencias de solución, o respondiendo a preguntas, algo que el candidato contrario efectuó con menor frecuencia; para la negativa solo existe una, la tipología de adversario (4\%).

Cuando se menciona los videos del candidato Arauz, cuyo mensaje era de esperanza, fueron aquellos que solventaban dudas que los usuarios dejaban en los comentarios de diversos videos. El candidato Arauz los respondía, mostrando soluciones o medidas que tomarían respecto a las actividades que los ciudadanos deseaban saber.

Gráfico 6. Cantidad y clasificación de mensajes con tipología asertiva y negativa del candidato Arauz. Elaboración propia a partir de datos de la ficha de observación.

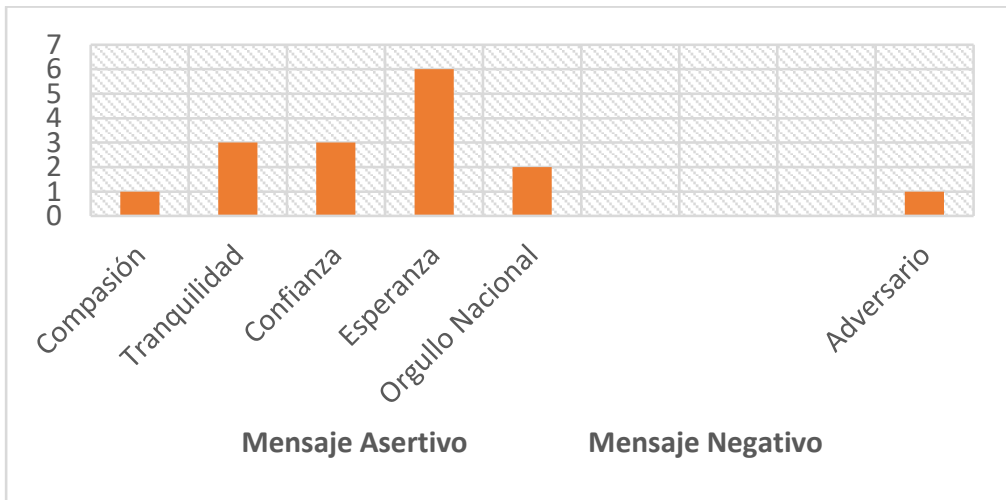

Cada video posee una descripción, esta fortalece y argumenta el mensaje emitido en el video. Es así, que cada descripción fue clasificada de acuerdo a la característica argumentativa que poseía (Gráfico 7). Por el candidato Lasso, la mayor parte de las descripciones de los videos ofrecen una promesa (28\%), seguida de descripciones cercanas $(20 \%)$, verdaderas $(20 \%)$ y que inciten a actuar (20\%). En último lugar quedan las descripciones atractivas (12\%). Por el contrario, se encuentra el candidato Arauz, quien la mayor parte de sus descripciones están equilibradas entre atractiva (25\%), cercana (25\%) y ofrecedoras de promesa (25\%), seguidas por descripciones verdaderas $(12,5$ $\%$ y que empujan a actuar (12,5\%). Es importante mencionar que un video del candidato Arauz no presentó descripción, por lo tanto, no se pudo analizar. 
Gráfico 7. Clasificación de las descripciones de los videos. Elaboración propia a partir de datos de la ficha de observación.

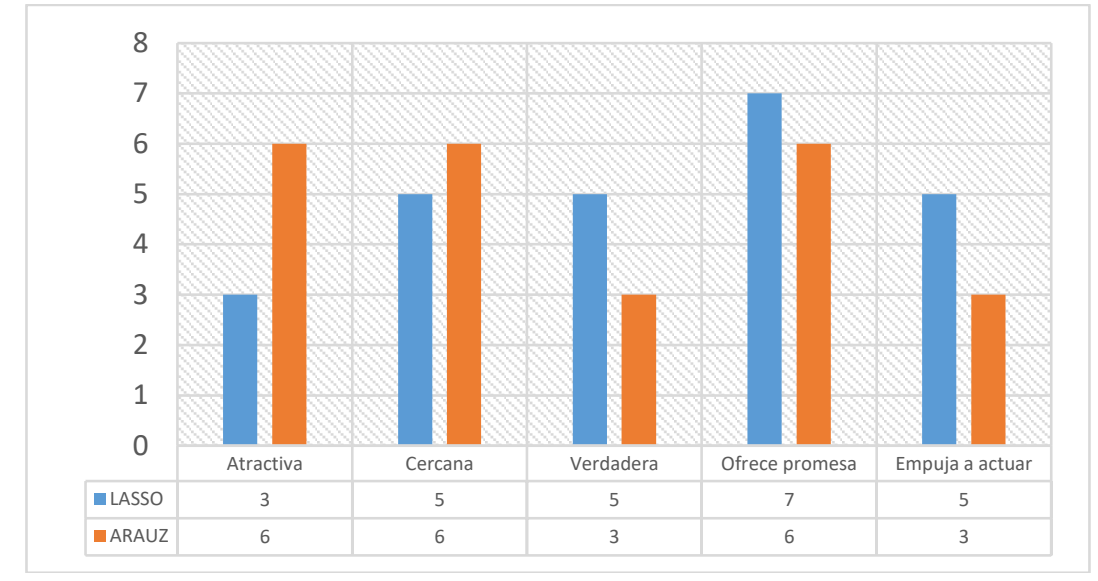

A través del análisis de cada descripción, y de los datos ofrecidos por la clasificación argumental podemos afirmar las siguientes ideas:

a) El candidato Arauz presenta descripciones mayormente elaboradas; pensadas no como refuerzo del mensaje del video, sino como añadidura, con algún dato extra que haga más interesante la visualización y atención del contenido. Esto hace que su descripción sea más atractiva y cercana.

b) El candidato Lasso presenta descripciones que se apegan al contenido emitido en los videos, y en varios de ellos, en donde el mensaje es claro, la descripción solo añade uno o dos hashtags de acompañamiento. Esto hace que sus descripciones sean vistas como menos atractivas, sin embargo, resultan más cercanas, esto como resultado del uso de palabras o mensajes comunes de fácil reconocimiento y recordación.

Cabe recalcar, que dentro de las descripciones se hace el uso de hashtags o etiquetas (Gráfico 8), estos son importantes porque además de fortalecer y acompañar un mensaje, ayudan a la distribución o segmentación del video. A través del registro, se evidenció que el hashtag más usado por el candidato Lasso es "\#LassoPresidente2021" (24\%), no obstante, este se encuentra inmerso en menos del $25 \%$ de los videos analizados. Un segundo hashtag fue "\#AndresNoMientasOtraVez" (8\%), mismo que se usó en los videos cuya temática principal era la de adversario, donde el candidato Lasso agregaba extractos de discursos del candidato opositor con el fin de llevar un mensaje 
negativo. Además, este último hashtag mencionado surgió en el debate presidencial, y desde allí fue usado como parte de una campaña por parte del candidato de CREO en contra del candidato de Unión por la Esperanza.

Por el contrario, existen cuatro hashtags que mayormente han sido usados en los videos del candidato Arauz: "\#AndresPresidente" (56 \%), "\#Ecuador" (56 \%), "\#ContigoConTodosAhora" (48 \%) y "\#MasAmorMenosHate" (44 \%); de los anteriormente mencionados, dos están inmersos en más del 50 \% de los videos, y los otros en más del $40 \%$, sirviendo como elementos de marca o de campaña electoral.

Por consiguiente, se afirma que el candidato Arauz mantuvo una línea de campaña definida de hashtags en la mayor cantidad de videos subidos, mientras que el candidato Lasso hizo uso de varios hashtags, no usando uno en específico como parte de su campaña, sino variando dependiendo el contenido y mensaje. Sin embargo, el usar una línea de campaña en específico no resultó favorable para el candidato Arauz, pues a pesar de aquello sus videos, en la mayoría, no sobrepasaban el millón de visualizaciones, caso opuesto al candidato Lasso, quien, a pesar de no usar una línea de hashtags, sus videos superan en un 50 $\%$ de visualizaciones y reacciones a los de Arauz.

Gráfico 8. Hashtags con mayor frecuencia de uso por los candidatos. Elaboración propia a partir de datos de la ficha de observación.

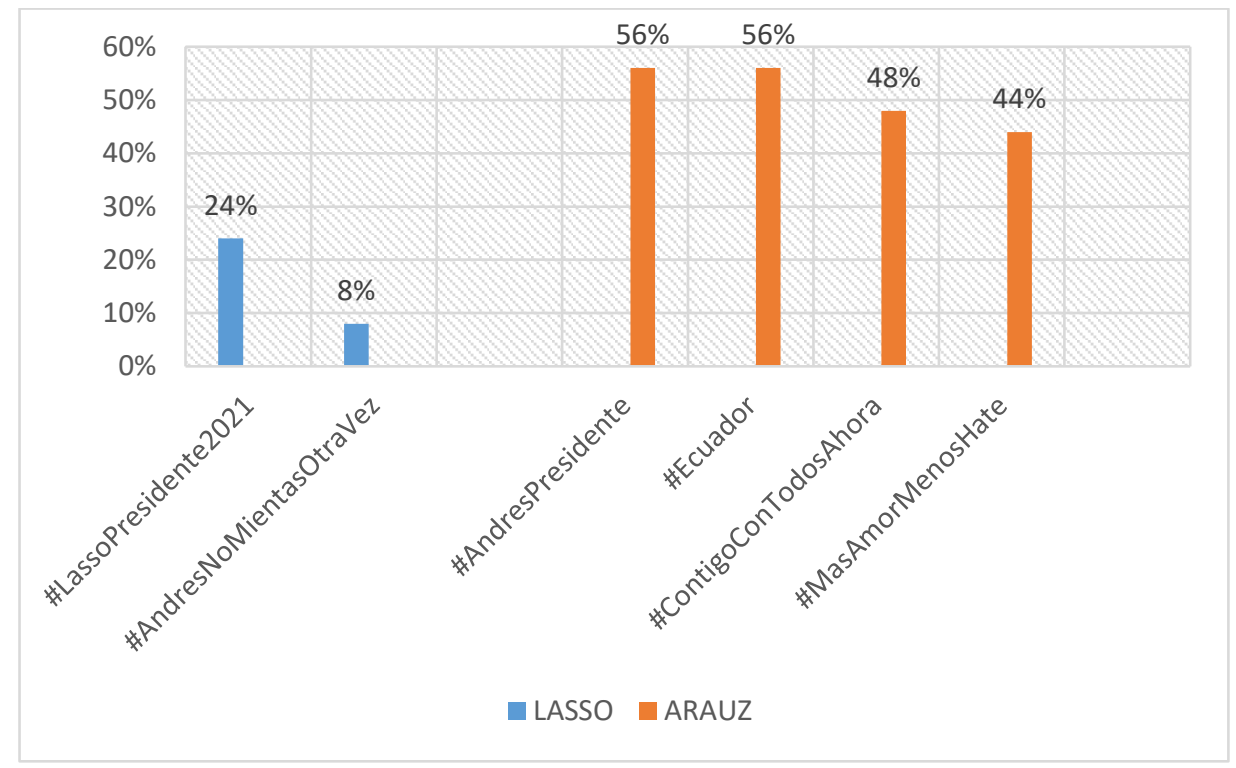


Un último elemento analizado, que también forma parte del discurso, es el uso de personajes. Es importante conocer quiénes son aquellos que intervienen en cada video, pues ayuda a los usuarios, ciudadanos a adquirir familiaridad al ver a otras personas confiar en un candidato. En los datos recolectados (Gráfico 9) se evidencia que, en ambos casos, cada candidato aparece en total de los videos propiamente analizados (100\%). Sin embargo, se registra un contraste de uso de recursos humanos a parte del candidato. Quien con mayor frecuencia participa e interactúa con más gente en sus videos es el candidato Lasso (más del $50 \%$ ), mientras que el candidato Arauz usa los videos como autopromoción, y son pocos los videos (menos del 50 \%) en que aparecen más personas.

Gráfico 9. Personajes que intervienen en los videos. Elaboración propia a partir de datos de la ficha de observación.

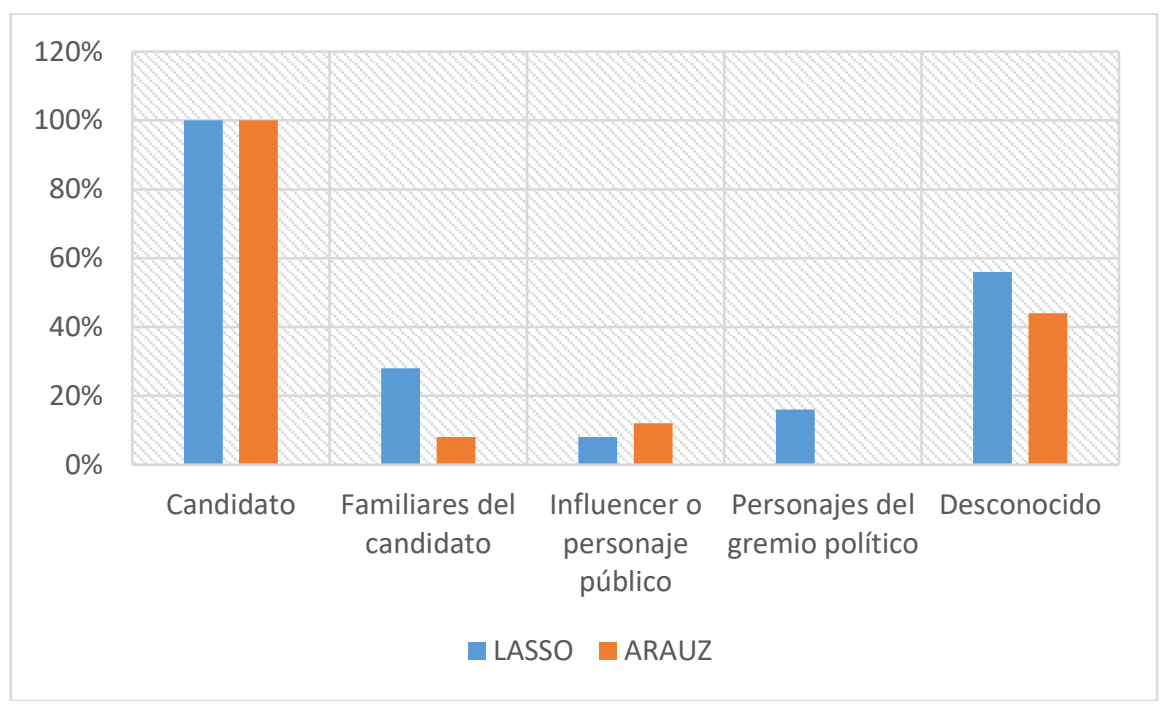

Tomando en cuenta todos los datos recolectados, queda evidenciado que el candidato con mayor presencia dentro de la red social estudiada fue Lasso, superando por mucho a Arauz. El contenido del candidato de CREO era variado, con contenidos creativos, innovadores, que llamaron la atención del público.

El uso de trends como formas de llevar un mensaje, de personajes con quienes la gente conectara, y de hashtags populares que los usuarios recordaran fácilmente, hizo que tuviera mayor aceptación, situación evidenciada en las estadísticas. Por otro lado, el candidato Arauz, a pesar de llevar con orden su campaña, con temáticas asertivas, y estrategias de posicionamiento, como los hashtags definidos, no logró que sus videos consiguieran el alcance esperado. 


\section{CONCLUSIONES}

Debido a la crisis sanitaria, el uso de los medios digitales se tornó fundamental. No se volvió una opción, sino una necesidad. La comunicación y transmisión de información a través de redes fue usada en todos los campos, incluyendo en la comunicación política.

La campaña por las elecciones presidenciales en Ecuador, que inicialmente surgió el 31 de diciembre de 2020, demuestra que la búsqueda de conectar con la mayor cantidad de audiencia posible, permitió que los candidatos hicieran uso de las redes sociales. El uso de TikTok, a pesar de que no fue usada como estrategia por el candidato Lasso en una primera vuelta, sí sucedió con el candidato Arauz. Sin embargo, en una segunda, viendo el alcance y conectividad que tuvieron otros candidatos, el representante de CREO optó por innovar y crear videos en la red social antes mencionada.

Definir una estrategia de discurso efectiva en TikTok resulta ser una prueba de ensayo y error, en vista de que a través de los datos recogidos se evidencia que cada video posee un propio alcance, sin importar si su mensaje es asertivo, negativo, informativo o reactivo, mientras que para el candidato Lasso el video con mayor alcance con temática asertiva, para el candidato Arauz fue con temática reactiva. No obstante, podemos afirmar que el uso de palabras comunes, hashtags del momento y personajes con quienes conectar a más del candidato, permitieron que una campaña resulte efectiva, algo evidenciado en la segunda vuelta de elecciones presidenciales realizadas en abril, donde el candidato Lasso fue proclamado presidente.

De la misma forma, se puede concluir es que el uso de la red social TikTok se tornó esencial para que los ciudadanos puedan conocer, a más de propuestas, la vida de cada candidato.

Es importante recalcar que el mostrar a TikTok como una herramienta principal de comunicación resulta ser una falacia, ya que, a pesar de tener un gran alcance de la audiencia, esta no representa un gran porcentaje de la población. Además, el intercambio de información es limitado a videos de 15, 60, y 180 segundos, sirviéndose más como un elemento publicitario y bloqueando una verdadera 
discusión de propuestas entre candidato-seguidor. Existen otras herramientas que si permiten dicha acción; TikTok sirve como un soporte informativo de propuestas, además de mostrar discusiones realizadas en reuniones con la comunidad, algo presentado en varios videos de los candidatos a quienes se realizó el estudio.

\section{Agradecimientos}

A Dios, quien es mi principal motivo y fortaleza. A mis padres y hermanas, por apoyarme en cada decisión. A mis docentes, y compañeros, quienes vieron mi crecimiento profesional y me motivaron cada día a no rendirme.

Un agradecimiento especial al Lcdo. Gerzon Cochea, quien fue pilar fundamental para la realización de este proyecto. Por su tiempo dedicado a la revisión, corrección y aprobación del mismo.

\section{REFERENCIAS}

[1] Peña E. Política 2.0: Cómo las Redes Sociales funcionan en la Política [Internet]. Bogotá: Universidad Cooperativa de Colombia; 2019 [Consultado 15 Jul 2021]. Disponible en: https://repository.ucc.edu.co/bitstream/20.500.12494/13501/1/2019_politi ca_redes_sociales.pdf

[2] Arbeláez M. Las tecnologías de la información y la comunicación (TIC) un instrumento para la investigación. Investig. andin. 2014; 16(29): p. 997 1000.

[3] Padilla EJ, Portilla GI, Torres-Mendoza M. Aprendizaje autónomo y plataformas digitales: el uso de tutoriales de YouTube de jóvenes en Ecuador. Estud. pedagóg. (Valdivia, En línea) [Internet] 2020 [Consultado 2021 Jul 20]; 46(2): 285-297. Disponible en: http://revistas.uach.cl/index.php/estped/article/view/6363 DOI 10.4067/S0718-07052020000200285

[4] Van-Djick J. La cultura de la conectividad: Una historia crítica de las redes sociales. Buenos Aires: Veintiuno Editores; 2016.

[5] De Salas M. La comunicación empresarial a través de internet. Primera ed. Valencia: Servicio de Publicaciones Universidad Cardenal HerreraCEU; 2002.

[6] Gelpi-Texeira R, Política 2.0: las redes sociales (Facebook y Twitter) como instrumento de comunicación política. Estudio: caso Uruguay [Internet]. 
Madrid: Universidad Complutense de Madrid; 2018 [Consultado 2021 Jul 17]. Disponible en: https://eprints.ucm.es/id/eprint/49515/1/T40361.pdf

[7] Gómez Castellanos RM, Ortiz Marín M, Concepción Montiel LE. Tecnologías de la comunicación y política 2.0. Espacios Públicos [Internet] 2011 [consultado 2021 Jul 17]; 14(30): p.72-84. Disponible en: https://www.redalyc.org/pdf/676/67618934006.pdf

[8] Bucheli K. Marketing político 2.0 y estrategias de la comunicación implementadas por el movimiento Alianza PAIS en la campaña electoral presidencial Ecuador año 2013. Quito: Pontificia Universidad Católica del Ecuador; 2014.

[9] Chapple C. Sensor Tower Blog. TikTok Crosses 2 Billion Downloads After Best Quarter For Any App Ever [Internet] San Francisco: 2020 Abr [Consultado 2021 Jul 10] Disponible en: https://sensortower.com/blog/tiktok-downloads-2-billion

[10] Mina A. ¿Comunicación Política en decadencia? ¿TikTok la nueva plataforma digital ayudará a conseguir simpatizantes? [Internet] Beers \& Politics; 2021 Ene 13. Disponible en: https://beersandpolitics.com/comunicacion-politica-en-decadencia-tiktokla-nueva-plataforma-digital-ayudara-a-conseguir-simpatizantes

[11] Tufiño N. Boa, Estrategia y Comunicación. Políticos de TikTok ¿una estrategia efectiva? [Internet] Quito: 2021 Feb 3. Disponible en: https://www.boa.ec/ultimo-post/elecciones-tiktok

[12] Garrido F. Comunicación Estratégica. España: Ediciones Gestión 2000; 2004.

[13] Capriotti P. Branding corporativo: Fundamentos para la gestión estratégica de la Identidad Corporativa. Santiago de Chile: Colección de Libros de la Empresa; 2009.

[14] Costa-Sánchez C, Piñeiro T. Estrategias de Comunicación Multimedia. Barcelona: Editorial UOC; 2013.

[15] Ugarte, D. El poder de las redes. Manual ilustrado para personas, colectivos y empresas abocados al ciberactivismo. Madrid: Ediciones El Cobre; 2007.

[16] Fog K, Budtz C, Munch P, Blanchette S. Storytelling: Branding in Practice. Segunda Edición. Dinamarca: Springer; 2010.

[17] Donstrup M. Propaganda en redes sociales: Análisis de contenido en Twitter durante la campaña electoral andaluza. Obra digit. [Internet]. 2019; 
(7):63-76.

Disponible

en: https://raco.cat/index.php/ObraDigital/article/view/363817/458081

[18] Martin J. La eficacia del Storytelling. MK: Marketing + Ventas. 2009; (251): p. 8-17.

[19] Hernández-Sampieri R, Fernández C, Baptista M. Metodología de la Investigación. Quinta ed. México: McGraw-Hill / Interamericana Editores S.A. de C.V.; 2010 